J. Austral. Math. Soc. 21 (Series A) (1976), 79-87.

\title{
BOUNDED INDEX AND SUMMABILITY METHODS
}

\author{
G. H. FRICKE AND R. E. POWELL
}

(Received 10 April 1974)

\section{Introduction and preliminary results}

An entire function $f(z)$ is of bounded index if there exists a non-negative integer $N$ such that

$$
\max _{0 \leqq j \leqq N}\left\{\frac{\left|f^{(j)}(z)\right|}{j !}\right\} \geqq \frac{\left|f^{(k)}(z)\right|}{k !} \text { for all } z \text { and all } k .
$$

The least such integer $N$ is called the index of $f$ (see Lepson (1966)).

A sequence $x=\left\{x_{k}\right\}_{0}^{x}$ of complex numbers is an entire sequence if $\sum_{k=0}^{x}\left|x_{k}\right| q^{k}$ converges for every positive integer $q$. If we denote by $\mathscr{E}$ the set of entire sequences, then we see that $\mathscr{E}$ can be identified with the class of entire functions. An entire sequence $x=\left\{x_{k}\right\}_{0}^{x}$ is of bounded index if $f(z)=\sum_{k=0}^{x} x_{k} z^{k}$ is an entire function of bounded index. We will denote the set of sequences of bounded index by $\mathscr{B}$. Furthermore, let $\ell$ be the set of all absolutely convergent sequences, that is, $\ell=\left\{x=\left\{x_{k}\right\}_{0}^{\infty}: \sum_{k=0}^{\infty}\left|x_{k}\right|<\infty\right\}$.

An infinite matrix $A=\left(a_{n, k}\right)$ of complex entries which transforms $\mathscr{E}$ into $\mathscr{E}$ $(\mathscr{B}$ into $\mathscr{B}, \ell$ into $\ell$ ) will be called an $\mathscr{E}-\mathscr{E}$ method $(\mathscr{B}-\mathscr{B}$ method, $\ell-\ell$ method).

In Fricke and Powell (1970), the authors have shown

Theorem 1. A matrix $A=\left(a_{n, k}\right)$ is an $\mathscr{E}-\mathscr{E}$ method if and only if for each integer $q>0$, there exist an integer $p>0$ and a constant $M>0$ such that

$$
\left|a_{n, k}\right| q^{n} \leqq M p^{k} \text { for all } n, k=0,1, \cdots \text {. }
$$

The Taylor matrix, $T(r)=\left(a_{n, k}\right)$, defined by

$$
a_{n, k}= \begin{cases}\left(\begin{array}{l}
k \\
n
\end{array}\right)(1-r)^{n+1} r^{k-n} & \text { if } k \geqq n \\
0 & \text { otherwise, }\end{cases}
$$

where $r$ is a complex number, is an $\mathscr{E}-\mathscr{E}$ method for any complex number $r$ (by Theorem 1). We now show: 
THEOREM 2. The Taylor matrix, $T(r)=\left(a_{n, k}\right)$, is a $\mathscr{B}-\mathscr{B}$ method for any complex number $r$.

PRoof. Let $x=\left\{x_{k}\right\}_{0}^{x} \in \mathscr{B}$, that is, $f(z)=\sum_{k=0}^{\infty} x_{k} z^{k}$ is a function of bounded index. Thus, for $y=\left\{y_{n}\right\}_{0}^{x}=A x$ (where $y_{n}=\sum_{k=0}^{x} a_{n, k} x_{k}$ ),

$$
\begin{aligned}
g(z)=\sum_{n=0}^{\infty} y_{n} z^{n} & =\sum_{n=0}^{\infty} \sum_{k=0}^{\infty} a_{n, k} x_{k} z^{n} \\
& =\sum_{n=0}^{\infty} \sum_{k=n}^{\infty}\left(\begin{array}{l}
k \\
n
\end{array}\right)(1-r)^{n+1} r^{k-n} x_{k} z^{n} \\
& =(1-r) \sum_{k=0}^{\infty} \sum_{n=0}^{k}\left(\begin{array}{l}
k \\
n
\end{array}\right)(1-r)^{n} z^{n} r^{k-n} x_{k} \\
& =(1-r) \sum_{k=0}^{\infty} x_{k}[(1-r) z+r]^{k} \\
& =(1-r) f([1-r] z+r) .
\end{aligned}
$$

From Fricke (to appear) we have that the class of functions of bounded index is closed under translation. Hence, $g(z)$ is of bounded index, that is,

$$
A x=y=\left\{y_{n}\right\}_{0}^{\infty} \in \mathscr{B} .
$$

It is readily seen that mere growth conditions on the entries of a matrix $A=\left(a_{n, k}\right)$ are not sufficient for $A$ to be a $\mathscr{B}-\mathscr{B}$ method. In fact, given any sequence $x=\left\{x_{k}\right\}_{0}^{x}$ of bounded index and any sequence $\left\{d_{n}\right\}_{0}^{x}$ of positive numbers, there exists a matrix $A=\left(a_{n, k}\right)$ with $a_{n, k}=0$ for $n \neq k$ and $\left|a_{n, n}\right| \leqq d_{n}$ for $n=0,1, \cdots$, such that $A x \notin \mathscr{B}$.

We now prove a result on functions of bounded index which we will require later on.

THeOREM 3. Let $f$ be a function of bounded index. If

$$
\lim _{n \rightarrow \infty} f^{(k)}\left(a_{n}\right)=0 \text { for } k=0,1, \cdots,
$$

where $\left\{a_{n}\right\}_{0}^{x}$ is a sequence of complex numbers, then, for all $r>0$,

$$
\lim _{n \rightarrow \infty} \max _{\left|z-a_{n}\right|=r}\left\{\left|f^{(k)}(z)\right|\right\}=0 \text { for all } k=0,1, \cdots \text {. }
$$

Proof. Let $f$ be of index $N$. It is sufficient to prove Theorem 3 for $r=1 /(2 N+2)$. Since $f$ is of index $N$ we have, for each $a_{n}$, that there exists an integer $\ell=\ell\left(a_{n}\right)$ with $0 \leqq \ell \leqq N$ such that

$$
\max _{\left|z-a_{n}\right|=r}\left\{\frac{\mid f^{(\ell)}(z)}{\ell !}\right\} \geqq \max _{\left|z-a_{n}\right|=r}\left\{\frac{\left|f^{(j)}(z)\right|}{j !}\right\} \text { for } j=0,1, \cdots .
$$

Hence, 


$$
\begin{aligned}
\max _{\left|z-a_{n}\right|=r}\left\{\frac{\left|f^{(\ell)}(z)\right|}{\ell !}\right\} & \geqq \max _{\left|r-a_{n}\right|=r}\left\{\frac{\mid f^{(\ell+b)}(z)}{(\ell+1) !}\right\} \\
& \geqq \frac{1}{(\ell+1) !} \frac{\max _{\left|z-a_{n}\right|=r}\left\{\left|f^{(\ell)}(z)\right|\right\}-\left|f^{(\ell)}\left(a_{n}\right)\right|}{r} \\
& \geqq 2\left(\max _{\left|z-a_{n}\right|=r}\left\{\frac{\left|f^{(\ell)}(z)\right|}{\ell !}\right\}-\frac{\left|f^{(\ell)}\left(a_{n}\right)\right|}{\ell !}\right) .
\end{aligned}
$$

Thus,

$$
\max _{\left|z-a_{n}\right|=r}\left\{\frac{\left|f^{(\ell)}(z)\right|}{\ell !}\right\} \leqq 2 \frac{\left|f^{(\ell)}\left(a_{n}\right)\right|}{\ell !}
$$

Therefore, for $n=0,1, \cdots$ and $j=0,1, \cdots$,

$$
\max _{\left|z-a_{n}\right|=r}\left\{\frac{\left|f^{(j)}(z)\right|}{j !}\right\} \leqq 2 \max _{0 \leq t \leq N}\left\{\frac{\left|f^{(t)}\left(a_{n}\right)\right|}{t !}\right\} \text {. }
$$

Thus, using the hypothesis,

$$
\lim _{n \rightarrow \infty} \max _{\left|z-a_{n}\right|=r}\left\{\left|f^{(j)}(z)\right|\right\}=0 .
$$

\section{The matrix $A\left(f, z_{i}\right)$}

For an entire function $f(z)$ and a sequence $\left\{z_{i}\right\}_{0}^{\infty}$ of complex numbers define the matrix transformation $A\left(f, z_{i}\right)=\left(a_{n, k}\right)$ by

$$
f(z)=\sum_{k=0}^{\infty} a_{n, k}\left(z-z_{n}\right)^{k} \text { for } n=0,1, \cdots
$$

For this matrix transformation we can express the Silvermann-Toeplitz conditions for regularity as follows.

Theorem 4. The matrix transformation $A\left(f, z_{i}\right)=\left(a_{n, k}\right)$ is regular if and only if

(i) $\lim _{n \rightarrow \infty} f^{(k)}\left(z_{n}\right)=0$ for $k=0,1, \cdots$,

(ii) $\lim _{n \rightarrow \infty} f\left(z_{n}+1\right)=1$,

and

(iii) $\sum_{k=0}^{\infty}\left|a_{n, k}\right| \leqq M$ for some $M>0$ and all $n=0,1, \cdots$

Proof. For the matrix $A\left(f, z_{i}\right)=\left(a_{n, k}\right)$, we have $a_{n, k}=\left(f^{(k)}\left(z_{n}\right)\right) / k$ ! for $n$, $k=0,1, \cdots$, and $f\left(z_{n}+1\right)=\sum_{k=0}^{\infty} a_{n, k}$. Hence, conditions (i), (ii) and (iii) are identical to the Silvermann-Toeplitz conditions 


$$
\begin{aligned}
& \lim _{n \rightarrow \infty} a_{n, k}=0 \text { for } k=0,1, \cdots, \\
& \lim _{n \rightarrow \infty} \sum_{k=1}^{x} a_{n, k}=1,
\end{aligned}
$$

and

$$
\sup _{n}\left\{\sum_{k=0}^{\infty}\left|a_{n, k}\right|\right\}<\infty \text {. }
$$

THEOREM 5. If $f(z)$ is an entire function of bounded index then $A\left(f, z_{i}\right)=\left(a_{n, k}\right)$ is not regular for any sequence $\left\{z_{i}\right\}_{0}^{\infty}$.

Proof. By Theorem 3, $\lim _{n \rightarrow \infty} f^{(k)}\left(z_{n}\right)=0$ for $k=0,1, \cdots$ implies $\lim _{n \rightarrow \infty} f\left(z_{n}+1\right)=0$ since $f$ is of bounded index. Thus, conditions (i) and (ii) of Theorem 4 cannot be satisfied simultaneously.

It is worth noting, however, that functions of bounded index can give rise to conservative matrices. For example, let $f(z)=e^{z}$ ( $f$ is of bounded index with index 0) and choose $z_{n}=2 \pi$ in. Thus

and

$$
\begin{aligned}
& f^{(k)}\left(z_{n}\right)=1 \text { for all } k, n=0,1, \cdots, \\
& f\left(z_{n}+1\right)=e \text { for all } n=0,1, \cdots, \\
& \sum_{k=0}^{x}\left|a_{n, k}\right|=e \text { for all } n=0,1, \cdots .
\end{aligned}
$$

We now examine the matrix $A^{\prime}\left(f, z_{i}\right)=\left(b_{n, k}\right)$ which is defined by $f(z)=$ $\sum_{n=0}^{\infty} b_{n, k}\left(z-z_{k}\right)^{n}$ for $k=0,1, \cdots$. The matrix $A^{\prime}\left(f, z_{i}\right)=\left(b_{n, k}\right)$ is the transpose of $A\left(f, z_{i}\right)=\left(a_{n, k}\right)$, that is, $a_{n, k}=b_{k, n}$ for $n, k=0,1, \cdots$.

THEOREM 6. If $f(z)$ is an entire function of bounded index then, for any sequence $\left\{z_{i}\right\}_{0}^{\infty}, A^{\prime}\left(f, z_{i}\right)=\left(b_{n, k}\right)$ is an $\ell-\ell$ method if and only if

$$
\sup _{n}\left\{\left|f^{(k)}\left(z_{n}\right)\right|\right\}<\infty \text { for } k=0,1, \cdots .
$$

Proof. Knopp and Lorentz (1949) showed that a necessary and sufficient condition for a matrix $A=\left(a_{n, k}\right)$ to be an $\ell-\ell$ method is

$$
\sup _{k}\left\{\sum_{n=0}^{\infty}\left|a_{n, k}\right|\right\}<\infty \text {. }
$$

Let $A^{\prime}\left(f, z_{i}\right)=\left(b_{n, k}\right)$ be an $\ell-\ell$ method. Thus, there exists a constant $M>0$ such that

$$
\sum_{n=0}^{\infty}\left|b_{n, k}\right| \leqq M \text { for } k=0,1, \cdots
$$

Hence,

$$
\left|b_{n, k}\right|=\frac{\left|f^{(n)}\left(z_{k}\right)\right|}{n !} \leqq M \text { for } n, k=0,1, \cdots
$$

Therefore, 


$$
\sup _{k}\left\{\left|f^{(n)}\left(z_{k}\right)\right|\right\} \leqq n ! M<\infty \text { for } k=0,1, \cdots \text {. }
$$

Now, let $f$ be an entire function of bounded index and let $\left\{z_{i}\right\}_{0}^{x}$ be a sequence such that

$$
\sup _{n}\left\{\left|f^{(k)}\left(z_{n}\right)\right|\right\}<\infty \text { for } k=0,1, \cdots .
$$

Since $f(z)$ is of bounded index we have that $f(2 z)$ is of bounded index (see Fricke (to appear)). Let $N$ be the index of $f(2 z)$. Thus,

$$
\max _{0 \equiv j \leq N}\left\{\frac{2^{j}\left|f^{(j)}(z)\right|}{j !}\right\} \geqq \frac{2^{n}\left|f^{(n)}(z)\right|}{n !} \text { for all } z \text { and all } n \text {. }
$$

Hence,

$$
\max _{0 \approx j \leq N}\left\{\frac{\left|f^{(j)}(z)\right|}{j !}\right\} \geqq 2^{n-N} \frac{\left|f^{(n)}(z)\right|}{n !} \text { for all } z \text { and all } n .
$$

Therefore,

$$
\begin{aligned}
\sum_{n=0}^{\infty}\left|b_{n, k}\right| & =\sum_{n=0}^{\infty} \frac{\left|f^{(n)}\left(z_{k}\right)\right|}{n !} \\
& \leqq \sum_{n=0}^{\infty} 2^{N-n} \max _{0 \leqq j \leqq N}\left\{\frac{\left|f^{(j)}\left(z_{k}\right)\right|}{j !}\right\} \\
& \leqq 2^{N+1} N ! \max _{0 \leqq j \equiv N}\left\{\left|f^{(j)}\left(z_{k}\right)\right|\right\}
\end{aligned}
$$

Now, since $\sup _{k}\left\{\max _{0 \leqq j \leqq N}\left(\left|f^{(j)}\left(z_{k}\right)\right|\right)\right\}<\infty$, we have $\sup _{k}\left\{\sum_{n=0}^{x}\left|b_{n, k}\right|\right\}<\infty$, which shows that $A^{\prime}\left(f, z_{i}\right)$ is an $\ell-\ell$ method.

THEOREM 7. If $f(z)$ is an entire function of bounded index, then for any sequence $\left\{z_{i}\right\}_{0}^{x}, A^{\prime}\left(f, z_{i}\right)=\left(b_{n, k}\right)$ is an $\mathscr{E}-\mathscr{E}$ method if and only if for each integer $n>0$ there exist an integer $p>0$ and a constant $M>0$ such that

$$
\left|f^{(n)}\left(z_{k}\right)\right| \leqq p^{k} M \text { for } k=0,1, \cdots \text {. }
$$

Proof. If $A^{\prime}\left(f, z_{i}\right)=\left(b_{n, k}\right)$ is an $\mathscr{E}-\mathscr{E}$ method then, by Theorem 1 , for each integer $q>0$, there exist an integer $t>0$ and a constant $T>0$ such that

$$
\left|b_{n, k}\right| q^{n} \leqq t^{k} T \text { for } n, k=0,1, \cdots \text {. }
$$

Thus, for $q=1$ there exist an integer $t$ and a constant $T$ such that

$$
\left|b_{n, k}\right|=\frac{\left|f^{(n)}\left(z_{k}\right)\right|}{n !} \leqq t^{k} T \text { for } n, k=0,1, \cdots \text {. }
$$

Hence, for each integer $n$ there exist an integer $p=t$ and a constant $M=n ! T$ such that

$$
\left|f^{(n)}\left(z_{k}\right)\right| \leqq t^{k} n ! T=p^{k} M \text { for } k=0,1, \cdots .
$$


Now, let $f(z)$ be an entire function of bounded index and let $\left\{z_{i}\right\}_{0}^{x}$ be a sequence such that for each integer $n>0$ there exist an integer $p>0$ and a constant $M>0$ such that

$$
\left|f^{(n)}\left(z_{k}\right)\right| \leqq p^{k} M \text { for } k=0,1, \cdots
$$

Let $N$ be the index of $f(z)$. Thus, there exist an integer $r>0$ and a constant $T$ such that, for $n \leqq N$,

$$
\left|f^{(n)}\left(z_{k}\right)\right| \leqq r^{k} T \text { for } k=0,1, \cdots .
$$

For an integer $q>0$ we have, by Fricke (to appear), $h(z)=f(q z)$ is of bounded index. Thus, for $q>0$, let $N_{q}$ be the index of $h(z)=f(q z)$, that is, for any $z \in \mathcal{V}$ and $j=0,1, \cdots$,

$$
\begin{aligned}
& \frac{\left\lfloor h^{(j)}(z) \mid\right.}{j !}=q^{\prime} \frac{\left|f^{(j)}(z)\right|}{j !} \\
& \leqq \max _{0 \leqq i \leq N_{q}}\left\{q^{i} \frac{\left|f^{(i)}(z)\right|}{i !}\right\} \\
& =\max _{0 \leqq i \leqq N_{q}}\left\{\frac{\left\lfloor\boldsymbol{h}^{(i)}(z) \mid\right.}{i !}\right\} \text {. }
\end{aligned}
$$

Hence, for $j=0,1, \cdots$,

$$
\begin{aligned}
& q^{j} \frac{\left|f^{(j)}\left(z_{k}\right)\right|}{j !} \leqq \max _{i \leqq N_{q}}\left\{q^{i} \frac{\left|f^{(i)}\left(z_{k}\right)\right|}{i !}\right\} \\
& \leqq q^{N_{a}} \max _{i \leqq N}\left\{\frac{\left|f^{(i)}\left(z_{k}\right)\right|}{i !}\right\} \\
& \leqq q^{N_{q}} \max _{i \leq N}\left\{\left|f^{(i)}\left(z_{k}\right)\right|\right\}
\end{aligned}
$$

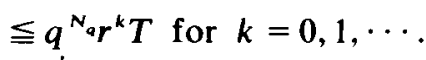

Therefore, for an integer $q>0$, there exist an integer $u=r$ and a constant $w=q^{N_{a}} T$ such that

$$
\left|b_{n, k}\right| q^{n}=\frac{\left|f^{(n)}\left(z_{k}\right)\right|}{n !} q^{n} \leqq q^{N_{a}} r^{k} T=u^{k} W \text { for } n, k=0,1, \cdots .
$$

Thus, by Theorem $1, A^{\prime}\left(f, z_{i}\right)$ is an $\mathscr{E}-\mathscr{E}$ method.

We now show that the condition that $f(z)$ is of bounded index cannot be omitted in Theorem 7.

THEOREM 8. There exists an entire function $f(z)$ of exponential type and of unbounded index and there exists a sequence $\left\{z_{i}\right\}_{0}^{x}$ such that for each integer $n$ there exist an integer $p$ and a constant $M$ with. 


$$
\left|f^{(n)}\left(z_{k}\right)\right| \leqq p^{n} M \text { for } k=0,1, \cdots,
$$

but $A^{\prime}\left(f, z_{i}\right)=\left(b_{n, k}\right)$ is not an $\mathscr{E}-\mathscr{E}$ method.

Proof. Let $\left\{a_{n}\right\}_{1}^{x}$ be a sequence of positive numbers such that $a_{1}=1$ and

$$
a_{k+1} \geqq \max \left\{3(k+1) a_{k}, a_{k} \frac{k+1}{k}\right\} \text { for } k=1,2, \cdots .
$$

S. M. Shah (1970) showed that $f(z)=\prod_{n=1}^{x}\left(1-z / a_{n}\right)^{n}$ is an entire function of exponential type and of unbounded index. Shah also showed that

$$
\lim _{n \rightarrow \infty} \frac{\left|f^{(n)}\left(a_{n}\right)\right|}{n !}=\infty \text {. }
$$

Therefore, there exists a sequence $\left\{\boldsymbol{n}_{k}\right\}_{k=1}^{x}$ such that

$$
\frac{\mid f^{\left(n_{k}\right)}\left(a_{n_{k}}\right)}{n_{k} !} \geqq k \text { ! for } k=1,2, \cdots .
$$

Choose the sequence $\left\{z_{k}\right\}_{k=0}^{x}$ by $z_{0}=1$ and $z_{k}=a_{n_{k}}$ for $k=1,2, \cdots$. Thus, for $f(z)=\prod_{n=1}^{x}\left(1-z / a_{n}\right)^{n}$ and the sequence $\left\{z_{k}\right\}_{0}^{\infty}$ we have $f^{(n)}\left(z_{k}\right)=0$ for $k>n$. Hence, for each integer $n$ there exist an integer $p=1$ and a constant $M=\max _{k \leq n}\left\{\left|f^{(n)}\left(z_{k}\right)\right|\right\}$ such that

$$
\left|f^{(n)}\left(z_{k}\right)\right| \leqq p^{k} M \text { for } k=0,1, \cdots .
$$

Now, for $A^{\prime}\left(f, z_{i}\right)=\left(b_{n, k}\right)$ and $k=1,2, \cdots$,

$$
\begin{aligned}
\left|b_{n_{k}, k}\right| & =\frac{\left|f^{\left(n_{k}\right)}\left(z_{k}\right)\right|}{n_{k} !} \\
& =\frac{\mid f^{\left(n_{k}\right)}\left(a_{n_{k}}\right)}{n_{k} !} \geqq k ! .
\end{aligned}
$$

Therefore, for any integer $r>0$ and any constant $T>0$, there exists $k_{0}$ such that

$$
\left|b_{n_{k}, k}\right| \geqq k !>r^{k} T \text { for } k>k_{0} .
$$

Thus, by Theorem $1, A^{\prime}\left(f, z_{i}\right)=\left(b_{n, k}\right)$ is not an $\mathscr{E}-\mathscr{E}$ method.

THEOREM 9. Let $f(z)$ be an entire function of bounded index and $\left\{z_{i}\right\}_{0}^{\infty}$ be a sequence of complex numbers. If either $A\left(f, z_{i}\right)=\left(a_{n, k}\right)$ or $A^{\prime}\left(f, z_{i}\right)=\left(b_{n, k}\right)$ is an $\ell-\ell$ method then $A^{\prime}\left(f, z_{i}\right)$ is an $\mathscr{E}-\mathscr{E}$ method.

PRoof. If either $A\left(f, z_{i}\right)$ or $A^{\prime}\left(f, z_{i}\right)$ is an $\ell-\ell$ method then either $\sum_{n=0}^{x}\left|a_{n, k}\right| \leqq M$, or

$$
\sum_{n=0}^{\infty}\left|b_{n . k}\right| \leqq M \text { for } k=0,1, \cdots
$$

Thus, either 


$$
\left|a_{n, k}\right|=\frac{\left|f^{(k)}\left(z_{n}\right)\right|}{k !} \leqq M,
$$

or

$$
\left|b_{n, k}\right|=\frac{\left|f^{(n)}\left(z_{k}\right)\right|}{n !} \leqq M \text { for } n, k=0,1, \cdots
$$

Hence,

$$
\frac{\left|f^{(n)}\left(z_{k}\right)\right|}{n !} \leqq M \text { for } n, k=0,1, \cdots
$$

Therefore, for each $n$ there exist $p=1$ and $T=n ! M$ such that

$$
\left|f^{(n)}\left(z_{k}\right)\right| \leqq n ! M=p^{k} T \text { for } k=0,1, \cdots .
$$

Thus, by Theorem $1, A^{\prime}\left(f, z_{i}\right)$ is an $\mathscr{E}-\mathscr{E}$ method.

\section{Application to analytic continuation}

Assume now that

$$
\lim _{n \rightarrow \infty} f^{(k)}\left(z_{n}\right)=0 \text { for } k=0,1, \cdots
$$

and

$$
\lim _{n \rightarrow \infty} f\left(z_{n}+1\right)=1
$$

Thus, for the matrix $A\left(f, z_{i}\right)=\left(a_{n . k}\right)$, we have

$$
\lim _{n \rightarrow \infty} a_{n . k}=0 \text { for } k=0,1, \cdots \text { and } \lim _{n \rightarrow \infty} \sum_{k=0}^{\infty} a_{n . k}=1 .
$$

THEOREM 10. The $A\left(f, z_{i}\right)$ transform continues the sequence of partial sums of the geometric series, $\Sigma_{k=0}^{\infty} z^{k}$, analytically into $\left\{z: \lim _{n \rightarrow \infty} f\left(z_{n}+z\right)=0\right\}$.

Proof. Let $A\left(f, z_{i}\right)=\left(a_{n, k}\right)$ and $\left\{S_{k}(z)\right\}_{0}^{\infty}$ be the sequence of $k$ th partial sums of geometric series. So, the $A\left(f, z_{i}\right)$ transform of $\left\{S_{k}(z)\right\}_{0}^{\infty}$ is given by

$$
\begin{aligned}
\sigma_{n}(z) & =\sum_{k=0}^{\infty} a_{n, k} S_{k}(z) \\
& =\sum_{k=0}^{\infty} a_{n, k} \frac{1-z^{k+1}}{1-z} \\
& =\frac{1}{1-z} \sum_{k=0}^{\infty} a_{n, k}-\frac{z}{1-z} \sum_{k=0}^{\infty} a_{n, k} z^{k} \\
& =\frac{1}{1-z} f\left(z_{n}+1\right)-\frac{z}{1-z} f\left(z_{n}+z\right) .
\end{aligned}
$$


So $\lim _{n \rightarrow \infty} \sigma_{n}(z)=\frac{1}{1-z} \Leftrightarrow \lim _{n \rightarrow \infty} f\left(z_{n}+z\right)=0$.

As an example, for Theorem 10, let $\alpha$ be a complex number with $\operatorname{Im} \alpha>0$, $f(z)=\exp \left\{i(z / \alpha)^{2}\right\}$, and $z_{n}=\alpha \sqrt{2 \pi n}-1$. Now, for fixed $k$,

$$
\lim _{n \rightarrow \infty}\left|f^{(k)}\left(z_{n}\right)\right|=\lim _{n \rightarrow \infty}\left|P_{k}\left(z_{n}\right)\right| \exp \left(\operatorname{Re}\left\{i\left(\frac{z_{n}}{\alpha}\right)^{2}\right\}\right)=0
$$

since $\lim _{n \rightarrow \infty} \exp \left(\operatorname{Re}\left\{i\left(z_{n} / \alpha\right)^{2}\right\}\right)=0$ and $P_{k}$ is a polynomial of degree $k$. Also, $\lim _{n \rightarrow \infty} f\left(z_{n}+1\right)=1$. Therefore,

$$
\left\{z: \lim _{n \rightarrow \infty} f\left(z_{n}+z\right)=0\right\}=\left\{z: \operatorname{Im}\left(\frac{z-1}{\alpha}\right)>0\right\},
$$

that is, the open half plane containing the origin whose boundary is $\{z: z-1=$ $\alpha x, x$ real\}. In particular, if $\alpha=i$ then $f(z)=\exp \left(-i z^{2}\right)$ and $z_{n}=\sqrt{2 \pi} n i-1$. Thus,

$$
\left\{z: \lim _{n \rightarrow \infty} f\left(z_{n}+z\right)=0\right\}=\{z: \operatorname{Re} z<1\}
$$

and, since $\{z:|z|<1\} \subseteq\left\{z: \lim _{n \rightarrow \infty} f\left(z_{n}+z\right)=0\right\}$, a minimal region into which the $A\left(f, z_{i}\right)$ transform provides the analytic continuation of an arbitrary Taylor series may be determined by use of the Okada theorem (Powell and Shah (1972; pages 155-162)).

\section{References}

G. H. Fricke (to appear), 'A characterization of functions of bounded index' .

G. H. Fricke and R. E. Powell (1970), 'A theorem on entire methods of summation', Comp. Math. 22, 253-259.

K. Knopp and G. G. Lorentz (1949), 'Beiträge zur absoluten Limitierung', Arch. Math. 2, 10-16.

B. Lepson (1966), Differential equations of infinite order, hyperdirichlet series and entire functions of bounded index. (Lecture notes, Summer Institute on Entire Functions, University of California, La Jolla).

R. E. Powell and S. M. Shah (1972), Summability Theory and Applications (London, Van Nostrand Reinhold, 1972).

S. M. Shah (1970), 'Entire functions of unbounded index and having simple zeros', Math. Z. 118, 193-196.

Wright State University

Dayton, Ohio, U.S.A.

Kent State University

Kent, Ohio, U.S.A. 